\title{
Time Reversal Aided Bidirectional OFDM Underwater Cooperative Communication Algorithm with the Same Frequency Transmission
}

\author{
Lingling Zhang, 1 Jianguo Huang, ${ }^{1}$ Chengkai Tang, ${ }^{2}$ and Houbing Song ${ }^{3}$ \\ ${ }^{1}$ School of Marine Science and Technology, Northwestern Polytechnical University, Shaanxi 710072, China \\ ${ }^{2}$ School of Electronics and Information, Northwestern Polytechnical University, Shaanxi 710072, China \\ ${ }^{3}$ Department of Electrical and Computer Engineering, West Virginia University, Montgomery, WV 25136, USA \\ Correspondence should be addressed to Lingling Zhang; zhanglingling9999@163.com
}

Received 8 January 2017; Revised 26 January 2017; Accepted 5 February 2017; Published 23 February 2017

Academic Editor: Fanli Meng

Copyright ( 2017 Lingling Zhang et al. This is an open access article distributed under the Creative Commons Attribution License, which permits unrestricted use, distribution, and reproduction in any medium, provided the original work is properly cited.

\begin{abstract}
In underwater acoustic channel, signal transmission may experience significant latency and attenuation that would degrade the performance of underwater communication. The cooperative communication technique can solve it but the spectrum efficiency is lower than traditional underwater communication. So we proposed a time reversal aided bidirectional OFDM underwater cooperative communication algorithm. The algorithm allows all underwater sensor nodes to share the same uplink and downlink frequency simultaneously to improve the spectrum efficiency. Since the same frequency transmission would produce larger intersymbol interference, we adopted the time reversal method to degrade the multipath interference at first; then we utilized the self-information cancelation module to remove the self-signal of OFDM block because it is known for sensor nodes. In the simulation part, we compare our proposed algorithm with the existing underwater cooperative transmission algorithms in respect of bit error ratio, transmission rate, and computation. The results show that our proposed algorithm has double spectrum efficiency under the same bit error ratio and has the higher transmission rate than the other underwater communication methods.
\end{abstract}

\section{Introduction}

Underwater communication, as one of the most challenging wireless communications, is of great difference compared to terrestrial wireless communication in system design. In underwater acoustic environment, the serious signal attenuation and severe reflection and diffusion result in short communication distance [1] and slow data rate [2]. Early underwater communications rely mainly on the increase of transmit power at underwater node to achieve long range signal transmission, but the requirement of large-scale power amplification equipment in this case limited the application in underwater mobile node [3]. With the increasing exploitation of marine resources, underwater communication is becoming more and more important. The underwater communication rate and the underwater communication distance seriously restrain the development of marine resources [4]. How to improve the underwater communication rate and increase underwater communication distance is the key problems that need to be solved in underwater communication nowadays.

An underwater communication system based on time domain channel equalization is presented in [5], which migrate the multipath induced intersymbol interference through adaptive equalization of time domain samples. To decrease the complexity for equalization in large latency underwater acoustic channel, frequency domain channel equalization is presented in [6], but both of these are insufficient to avoid the short range issue owing to the serious attenuation. To this end, underwater cooperative communication method is proliferated to extend the communication distance, in which data hops from the source to destination through several relays. However, it leads to more serious multipath effect, which results in high bit error rate. An underwater acoustic cooperative communication based on LDPC coding proposed in [7] can decrease the bit error rate, but with high complexity. 
An underwater acoustic cooperative communication based on frequency domain multiple access is supposed to increase the data rate by allowing several sensors to transmit signals simultaneously, but since the underwater acoustic channel is bandwidth limited, the performance is unsatisfactory [8]. Most underwater acoustic cooperative communications adopt time domain multiple access, which allows only one task in each time slot; therefore it leads to poor spectrum efficiency [9]. To this end, this paper presents a new underwater acoustic cooperative communication method based on time reversal and self-interference suppression in the same frequency. In this network, cooperative nodes share the common uplink and downlink frequency and transmit to the central base station simultaneously, to realize spectrum resource multiplexing. Moreover, the time reversal technology is adopted at the base station to migrate multipath interference. Furthermore, using the acknowledged information of selfsignal, self-interference is suppressed in the forwarded signal from the base station. In this network, the spatial processing requirement of the cooperative nodes is quite low; therefore, the size can be compact. In addition, OFDM modulation is adopted to decrease the complexity of channel equalization and frequency converter. This structure is available to current underwater detection and communication network.

\section{Bidirectional Underwater Communication System Model}

The complex underwater environment causes kinds of interferences in the communication channels, most of which attributes to multipath interference and signal attenuation. The structure of the self-interference suppressed time reversal cooperative transmission system is shown in Figure 1.

Assume the distance between these two sensor nodes is quite far; then because of the severe attenuation over long range underwater acoustic channel, the base station is required for relaying to enable the bidirectional transmission [10]. In Figure 1, taking into account the fact that the size of the sensor nodes is often limited, it supposes only one transmitter and receiver elements are available at each node. As the core of this cooperative network, there are $N_{R}$ pairs of transmitter and receiver elements at the Underwater Base Station, with each pair locating at the same position. Group the transmitted information of each sensor node to blocks of length $N_{a}$, and denote the $n_{b}$ th information block of the $n_{t}$ th sensor node which is

$$
\begin{aligned}
\mathbf{a}_{n_{t}} & \left(n_{b}\right) \\
\quad & =\left[a_{n_{t}}\left(n_{b}, 0\right), a_{n_{t}}\left(n_{b}, 1\right), \ldots, a_{n_{t}}\left(n_{b}, N_{a}-1\right)\right]^{T},
\end{aligned}
$$

where $a_{n_{t}}\left(n_{b}, 0\right) \in\{0,1\}$ and $j=0,1, \ldots, N_{a}-1$. Encode the information block with coding rate $R_{c}$; then we could get the encoded bits with block length $N=N_{a} / R_{c}$ as

$$
\begin{aligned}
\mathbf{b}_{n_{t}} & \left(n_{b}\right) \\
\quad & =\left[b_{n_{t}}\left(n_{b}, 0\right), b_{n_{t}}\left(n_{b}, 1\right), \ldots, b_{n_{t}}\left(n_{b}, N-1\right)\right]^{T} .
\end{aligned}
$$

In order to avoid the burst error and decrease the correlation between adjacent bits, interleave the encoded bits as

$$
\mathbf{c}_{n_{t}}\left(n_{b}\right)=\left[c_{n_{t}}\left(n_{b}, 0\right), c_{n_{t}}\left(n_{b}, 1\right), \ldots, c_{n_{t}}\left(n_{b}, N-1\right)\right]^{T} .
$$

Conversing these bits from serial to parallel with length $Q=\log _{2} M$, we can obtain $K_{d}$ vectors, and (3) could be represented as

$$
\mathbf{c}_{n_{t}}\left(n_{b}\right)=\left[\mathbf{c}_{n_{t}, 0}^{T}\left(n_{b}\right), \mathbf{c}_{n_{t}, 1}^{T}\left(n_{b}\right), \ldots, \mathbf{c}_{n_{t}, K_{d}-1}^{T}\left(n_{b}\right)\right]^{T}
$$

where $K_{d}=N / Q$, and $k_{d}\left(k_{d}=0,1, \ldots, K_{d}-1\right)$ th vector is

$$
\begin{gathered}
\mathbf{c}_{n_{t}, k_{d}}\left(n_{b}\right)=\left[c_{n_{t}}\left(n_{b}, k_{d} Q\right), c_{n_{t}}\left(n_{b}, k_{d} Q+1\right), \ldots,\right. \\
\left.c_{n_{t}}\left(n_{b}, k_{d} Q+Q-1\right)\right]^{T} .
\end{gathered}
$$

Map this vector according to Mary order PSK as $m_{n_{t}}\left(n_{b}, k_{d}\right)=\alpha_{m} \in \mathcal{S}$, with the alphabet

$$
\mathcal{S}=\left\{\alpha_{0}, \alpha_{1}, \ldots, \alpha_{M-1}\right\}
$$

and the corresponding vector pattern is $\mathbf{c}_{m}=\left[c_{m, 0}, c_{m, 1}, \ldots\right.$, $\left.c_{m, \mathrm{Q}-1}\right]^{T}$. Therefore, the transmitted symbol within the $n_{b}$ th block of the $n_{t}$ th sensor node is

$$
\begin{aligned}
m_{n_{t}} & \left(n_{b}\right) \\
& =\left[m_{n_{t}}\left(n_{b}, 0\right), m_{n_{t}}\left(n_{b}, 1\right), \ldots, m_{n_{t}}\left(n_{b}, K_{d}-1\right)\right]^{T} .
\end{aligned}
$$

Multiplexing these symbols to orthogonal frequency bins, the obtained OFDM signal is represented as

$$
\begin{aligned}
& x_{n_{t}}(t)=\Re\left\{\left[\sum_{n_{b}} \sum_{k} d_{n_{t}}\left(n_{b}, k\right) e^{j 2 \pi k \Delta f t} g^{n_{t}}\left(t-n_{b} T_{b}\right)\right]\right. \\
& \left.\cdot e^{j 2 \pi f_{0} t}\right\} \\
& =\Re\left\{\left[\sum_{n_{b}} \sum_{k \in \Theta_{a}^{n_{t}}} d_{n_{t}}\left(n_{b}, k\right) e^{j 2 \pi k \Delta f t} g^{n_{t}}\left(t-n_{b} T_{b}\right)\right]\right. \\
& \left.\cdot e^{j 2 \pi f_{0} t}\right\},
\end{aligned}
$$

where $k$ denotes the index of the subcarrier, with the range $k \in\{0, K-1\}, K$ denotes the total number of subcarriers, $\Delta f$ denotes the subcarrier spacing, $f_{0}$ denotes the starting frequency of the uplink transmission, and $g(t)$ denotes the pulse shaping. Aiming at measuring the noise level or preventing frequency leakage, null subcarriers of the $n_{t}$ th user node in the $n_{b}$ th block are assigned as $d^{n_{t}}\left(n_{b}, k\right)=0$ for $k \in \Theta_{\text {null }}^{n_{t}}$. Denote $\Theta_{a}^{n_{t}}$ as the active subcarrier set of the $n_{t}$ th user node; we have $\Theta_{a}^{n_{t}} \cup \Theta_{\text {null }}^{n_{t}}=[0, K-1]$ and $\Theta_{a}^{n_{t}} \cap \Theta_{\text {null }}^{n_{t}}=\emptyset$. The active subcarriers are categorized into $K_{p}$ pilot subcarriers and $K_{d}$ data subcarriers, with $d^{n_{t}}\left(n_{b}, k\right)=m^{n_{t}}\left(n_{b}, k_{d}\right)$ if $k \in \Theta_{\text {data }}^{n_{t}}$, 


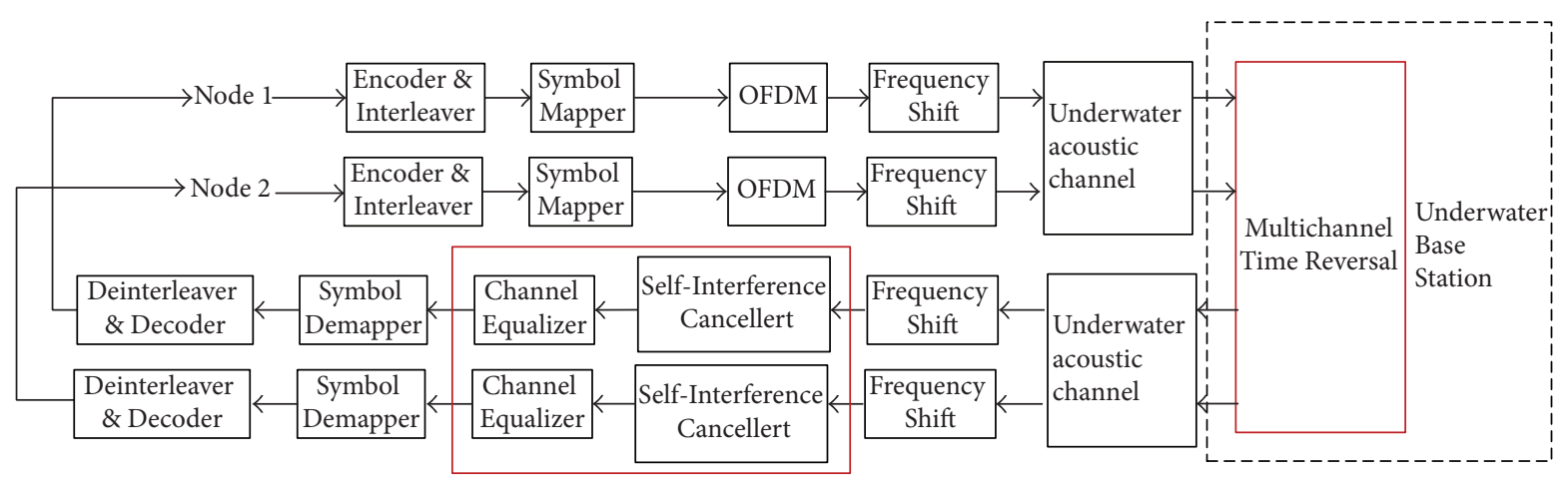

FIGURE 1: Underwater cooperative transmission system structure.

while $d^{n_{t}}\left(n_{b}, k\right)=p^{n_{t}}\left(n_{b}, k_{d}\right)$ if $k \in \Theta_{\text {pilot }}^{n_{t}}$. To avoid interference, set $\Theta_{\text {pilot }}^{n_{t}} \cup \Theta_{\text {data }}^{n_{t}}=\Theta_{a}^{n_{t}}$ and $\Theta_{\text {pilot }}^{n_{t}} \cap \Theta_{\text {data }}^{n_{t}}=\emptyset$.

At the $n_{r}$ th receiver element of the base station, it collects signals from all user nodes as

$$
y_{n_{r}}(t)=\sum_{n_{t}} \sum_{p} h_{p}^{n_{r}, n_{t}} x_{n_{t}}\left(t-\tau_{p}\right)+\xi_{n_{r}}(t)
$$

where $p$ denotes the path index, with $h_{p}$ and $\tau_{p}$ denoting the amplitude and time delay of the corresponding path, respectively. For description brevity, all time delay has been mapped to grid, inserting some zeros in $h_{p}^{n_{r}, n_{t}}$ artificially to ensure the delay of the $p$ th path of any arbitrary transceiver pair to be $\tau_{p}$. The received ocean noise at the $n_{r}$ th element of the base station denoted as $\xi_{n_{r}}(t)$ is assumed to be additive.

\section{Time-Reverse-and-Forward Module}

Assume the amplitude and time delay of the underwater acoustic channel are constant and reciprocal in a certain interval [11-13]; that is to say, the downlink from the $n_{r}$ th element of the base station to the $n_{t}$ th user node is assumed to be the same as the uplink from the $n_{t}$ th user node to the $n_{r}$ th element of the base station. On this basis, to reduce the processing complexity at the base station and improve transmission efficiency, rather than demodulating the received signal at the base station, it convolutes the received signal with time reversed channel response and forwards the mixture signal to all user nodes. In this case, the signal arrived at the $n_{t}$ th user node is

$$
q_{n_{t}}(t)=\sum_{n_{t}} \sum_{p} h_{p}^{n_{r}, n_{t}} z_{n_{r}}\left(t-\tau_{p}\right)+w_{n_{t}}(t)
$$

Since the frequency is shared by all user nodes, the mixture signal $z_{n_{r}}(t)$ includes the information from all user nodes. Therefore, the received signal at each user node includes not only information from other user nodes, but also self-information backpropagation, in addition to the serious multipath effect, which rises great challenge to bidirectional cooperative communication. Limited by the size of the user nodes, only single transmitter element and receiver element are deployed; thus it is hard to eliminate the multipath interference and self-information interference with single channel.

In a two-user nodes scenario as shown in Figure 1, we aim to solve the multipath interference and self-information interference, adopt the time reversal processing at the base station, and shift the frequency to downlink frequency as

$$
z_{n_{r}}(t)=\left(y_{n_{r}}(t) \otimes h^{n_{r}, 1}(-t) \otimes h^{n_{r}, 2}(-t)\right) e^{j 2 \pi\left(f_{2}-f_{1}\right) t},
$$

where $f_{2}$ denotes the central downlink frequency and $f_{1}=$ $f_{0}+K / 2 * \Delta f$ denotes the central uplink frequency. Uplink and downlink transmit simultaneously with this frequency division scheme, rather than partitioning different frequency to each user node, since it is easy to expand this cooperative transmission to more user nodes, even though the bandwidth of underwater acoustic channels is quite limited.

In the bidirectional cooperative transmission, the equivalent channel filter experienced by the signal transmitted by user 1 through the $n_{r}$ th element of the base station to itself is described as

$$
v_{11}^{n_{r}}=h^{n_{r}, 1}(t) \otimes h^{n_{r}, 1}(-t) \otimes h^{n_{r}, 2}(-t) \otimes h^{n_{r}, 1}(t) .
$$

The equivalent channel filter experienced by the signal transmitted by user 1 through the $n_{r}$ th element of the base station to user 2 is described as

$$
v_{12}^{n_{r}}=h^{n_{r}, 1}(t) \otimes h^{n_{r}, 1}(-t) \otimes h^{n_{r}, 2}(-t) \otimes h^{n_{r}, 2}(t) .
$$

The equivalent channel filter experienced by the signal transmitted by user 2 through the $n_{r}$ th element of the base station to user 1 is described as

$$
v_{21}^{n_{r}}=h^{n_{r}, 2}(t) \otimes h^{n_{r}, 1}(-t) \otimes h^{n_{r}, 2}(-t) \otimes h^{n_{r}, 1}(t) .
$$

The equivalent channel filter experienced by the signal transmitted by user 2 through the $n_{r}$ th element of the base station to itself is described as

$$
v_{22}^{n_{r}}=h^{n_{r}, 2}(t) \otimes h^{n_{r}, 1}(-t) \otimes h^{n_{r}, 2}(-t) \otimes h^{n_{r}, 2}(t) .
$$


Therefore, the received signal at user node 1 is written as

$$
\begin{aligned}
q_{1}(t)= & \sum_{n_{r}} x_{2}(t) e^{j 2 \pi\left(f_{2}-f_{1}\right) t} \otimes v_{21}^{n_{r}}(t) \\
& +\sum_{n_{r}} x_{1}(t) e^{j 2 \pi\left(f_{2}-f_{1}\right) t} \otimes v_{11}^{n_{r}}(t)+w_{1}^{\prime}(t) \\
= & \underbrace{x_{2}(t) e^{j 2 \pi\left(f_{2}-f_{1}\right) t} \otimes v_{21}(t)}_{q_{1 s}(t)} \\
& +\underbrace{x_{1}(t) e^{j 2 \pi\left(f_{2}-f_{1}\right) t} \otimes v_{11}(t)}_{q_{1 i}(t)}+\underbrace{w_{1}^{\prime}(t)}_{q_{1 n}(t)} .
\end{aligned}
$$

Likewise, the received signal at user node 2 is written as

$$
\begin{aligned}
q_{2}(t)= & \underbrace{x_{1}(t) e^{j 2 \pi\left(f_{2}-f_{1}\right) t} \otimes v_{12}(t)}_{q_{2 s}(t)} \\
& +\underbrace{x_{2}(t) e^{j 2 \pi\left(f_{2}-f_{1}\right) t} \otimes v_{22}(t)}_{q_{2 i}(t)}+\underbrace{w_{2}^{\prime}(t)}_{q_{2 n}(t)},
\end{aligned}
$$

where $q_{n_{t} s}$ denotes the desirable signal from the other user node to the $n_{t}$ th user node, while $q_{n_{t} i}$ denotes the unwanted interference from itself. According to the spatial reciprocity of the channel, $q_{n_{t} s}$ is temporal and spatial focusing of the desirable signal, and the equivalent channel is represented as

$$
\begin{aligned}
& v_{11}(t)=\sum_{n_{r}} v_{11}^{n_{r}}(t), \\
& v_{12}(t)=\sum_{n_{r}} v_{12}^{n_{r}}(t), \\
& v_{21}(t)=\sum_{n_{r}} v_{21}^{n_{r}}(t), \\
& v_{22}(t)=\sum_{n_{r}} v_{22}^{n_{r}}(t) .
\end{aligned}
$$

According to the underwater physics, the equivalent channel is a $q$-function, and it approaches a Dirac delta function in ideal case.

\section{Self-Interference Cancelation Method}

At the receiver end of the $n_{t}$ th user node, downshift the frequency to baseband firstly, and then isolate the received signal from its self-information interference, since self-signal is the main contribution in the interference. The self-information interference cancelation method is shown in Figure 2.

In ideal case, after self-information cancelation, the residue signal $r_{n_{t}}(t)$ is proportion to the transmitted signal from the other user node. Therefore, the estimation of selfinformation interference is represented as

$$
\widehat{q}_{n_{t} n}(t)=\sum_{i} c_{i} x_{n_{t}}(t-\tau-i)
$$

where $\tau$ denotes the delay of the channel filter, and the coefficients of the cancelation are updated by

$$
c_{i}(n+1)=c_{i}(n)+\eta e^{*}(n) x_{n_{t}}(t-\tau-i),
$$

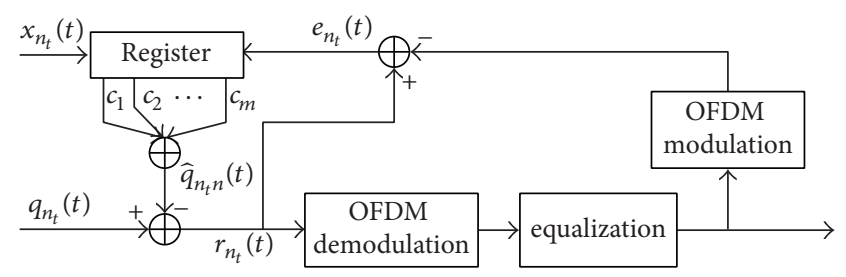

FIGURE 2: Underwater cooperative transmission system structure.

where $\eta$ denotes the update step, and the residue signal is represented as

$$
r_{n_{t}}(t)=q_{n_{t}}(t)-\widehat{q}_{n_{t} n}(t)
$$

Transform the residue signal into frequency domain; then we have

$$
\mathbf{R}_{n_{t}}=\mathbf{V}_{n_{t}, n_{t}^{\prime}} \mathbf{d}_{n_{t}^{\prime}}+\mathbf{W}_{n_{t}}
$$

According to the linear minimum mean square error criterion, the desired transmitted symbol estimated at the user node $n_{t}$ is denoted as

$$
\widehat{\mathbf{d}}_{n_{t}^{\prime}}=\overline{\mathbf{d}}_{n_{t}^{\prime}}+\mathscr{C}_{\mathbf{R}_{n_{t}} \mathbf{d}_{n_{t}^{\prime}}} \mathscr{C}_{\mathbf{R}_{n_{t}}}^{-1} \mathbf{R}_{n_{t}}\left(\mathbf{R}_{n_{t}}-\mathbf{V}_{n_{t}, n_{t}^{\prime}} \mathbf{d}_{n_{t}^{\prime}}\right)
$$

where $\overline{\mathbf{d}}_{n_{t}^{\prime}}$ denotes the expectation of the transmitted symbols, $\overline{\mathbf{R}}_{n_{t}}$ denotes the expectation of the received symbols, $\mathscr{C}_{\mathbf{R}_{n_{t}}} \mathbf{d}_{n_{t}^{\prime}}$ denotes the covariance matrix of the transmitted symbols and received symbols as

$$
\mathscr{C}_{\mathbf{R}_{n_{t}} \mathbf{d}_{n_{t}^{\prime}}}=E\left[\left(\mathbf{d}_{n_{t}^{\prime}}-\overline{\mathbf{d}}_{n_{t}^{\prime}}\right)\left(\mathbf{R}_{n_{t}}-\overline{\mathbf{R}}_{n_{t}}\right)^{H}\right]=\boldsymbol{\Phi}_{n_{t}^{\prime}} \mathbf{V}_{n_{t}, n_{t}^{\prime}}^{H}
$$

and $\mathscr{C}_{\mathbf{R}_{n_{t}} \mathbf{R}_{n_{t}}}$ denotes the autocovariance of the received symbols as

$$
\mathscr{C}_{\mathbf{R}_{n_{t}} \mathbf{R}_{n_{t}}}=\mathbf{V}_{n_{t}, n_{t}^{\prime}} \boldsymbol{\Phi}_{n_{t}^{\prime}} \mathbf{V}_{n_{t}, n_{t}^{\prime}}^{H}+\sigma_{\eta}^{2},
$$

where $\Phi_{n_{t}^{\prime}}$ denotes the autocovariance of the transmitted symbols

$$
\boldsymbol{\Phi}_{n_{t}^{\prime}}=E\left[\left(\mathbf{d}_{n_{t}^{\prime}}-\overline{\mathbf{d}}_{n_{t}^{\prime}}\right)\left(\mathbf{d}_{n_{t}^{\prime}}-\overline{\mathbf{d}}_{n_{t}^{\prime}}\right)^{H}\right]
$$

Suppose the transmitted symbols on different frequency bins are dependent on each other; thus

$$
\boldsymbol{\Phi}_{n_{t}^{\prime}}=\operatorname{diag}\left\{\sigma_{n_{t}^{\prime}}^{2}(0), \sigma_{n_{t}^{\prime}}^{2}(1), \ldots, \sigma_{n_{t}^{\prime}}^{2}(K-1)\right\} .
$$

At the beginning, since no prior information is available, the estimation symbols are represented as

$$
\widehat{\mathbf{d}}_{n_{t}^{\prime}}=\mathbf{V}_{n_{t}, n_{t}^{\prime}}^{H}\left(\mathbf{V}_{n_{t}, n_{t}^{\prime}} \mathbf{V}_{n_{t}, n_{t}^{\prime}}^{H}+\sigma_{\eta}^{2} \mathbf{I}\right)^{-1} \mathbf{R}_{n_{t}}
$$

\section{Simulation and Analysis}

According to the statistical analysis of lake channel, a sparse and spatial reciprocal channel is adopted to simulate the 


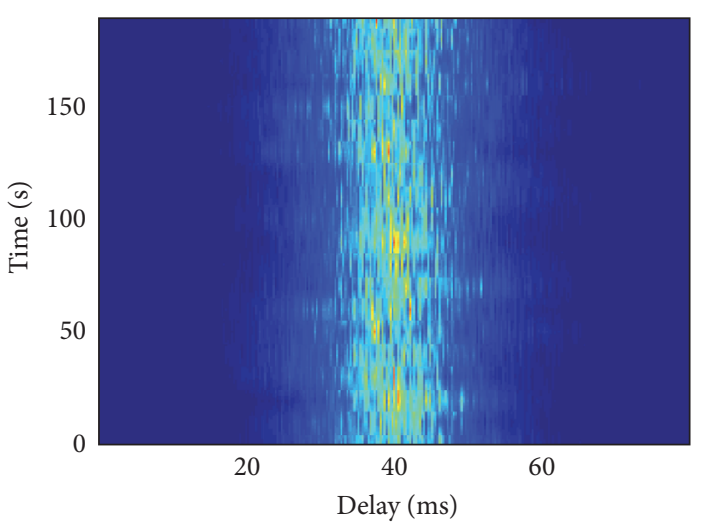

(a) $v_{11}$

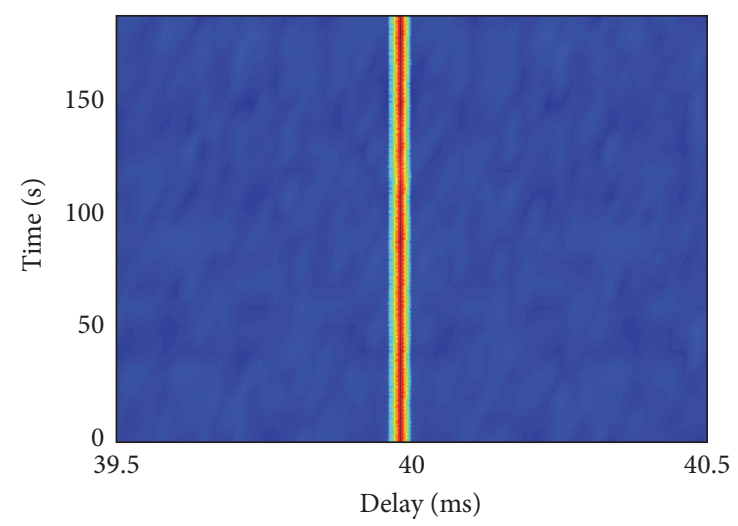

(c) $v_{21}$

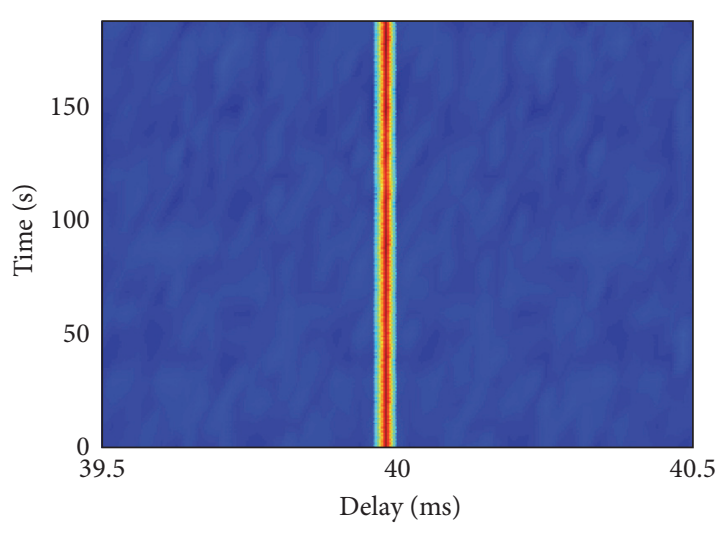

(b) $v_{12}$

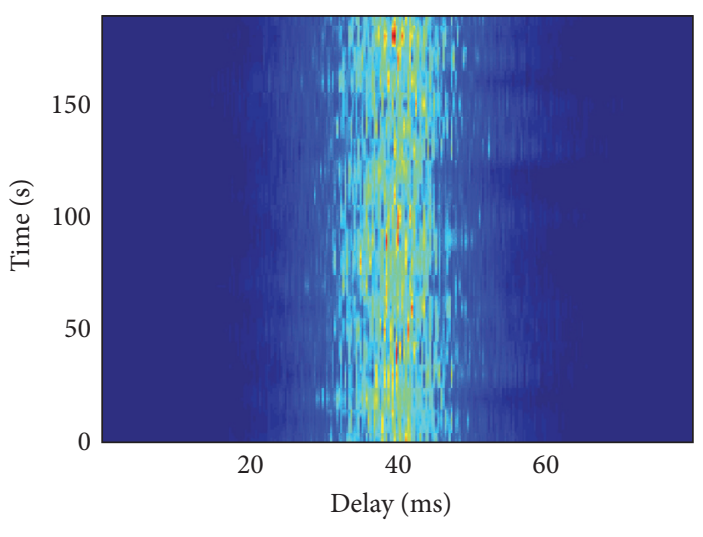

(d) $v_{22}$

FIGURE 3: The equivalent channel filter in time-reverse-and-forward underwater acoustic cooperative communication.

underwater acoustic channel [14]. The cooperative network includes two user nodes and one base station. Each user node deploys single transmitter and receiver. The base station consists of 16 transmitters and receivers. The channel between each user node to any element of the base station is independent of other and considers each as a six paths' random walk process with mean delay interval of $3 \mathrm{~ms}$. The amplitude is constant with each OFDM block and follows Gaussian distribution with initial exponent power delay profile. At the transmitter of each user node, the data is encoded by $\left(3,\left[\begin{array}{ll}5 & 7\end{array}\right]\right)$ convolution encoder and randomly interleaved; then QPSK constellation is mapped to form blocks of OFDM symbols.

5.1. Channel Interference Analysis. To evaluate the channel interference suppression performance, the time evolution of the equivalent channel filter from the source user node to the end user node in time-reverse-and-forward underwater acoustic cooperative communication is shown in Figure 3. For comparison, under the same channel condition, the time evolution of the equivalent channel filter from the source user node to the end user node in amplify-and-forward underwater acoustic cooperative communication is shown in Figure 4. From Figures 3 and 4, we can see that the channel is compressed in time-reverse-and-forward underwater acoustic cooperative communication but dispersed in amplifyand-forward underwater acoustic cooperative communication. Therefore, spatial diversity gain is easier to collect in time-reverse-and-forward underwater acoustic cooperative communication.

5.2. Bit Error Ratio Performance. In the bidirectional cooperative communication, spatial focusing is achieved by timereverse-and-forward for the desired signal transmission direction. However, since the equivalent channel is a $q$ function, rather than an ideal Dirac delta function, the communication performance degrades due to the self-information interference [16]. Therefore, the bit error ratio performance of time-reverse-and-forward underwater acoustic cooperative communication with respect to different numbers of taps of additional self-interference cancelation is shown in Figure 5. From Figure 5, it can be seen that, with small numbers of taps, the bit error ratio is still large, since the residue self-information interference cannot be well eliminated. With the increasing of the number of taps, the bit error ratio is decreasing and reaches $10^{-3}$ with 11 taps of self-interference cancelation while SNR is at $19 \mathrm{~dB}$. When the number of taps is over 13 , the performance reaches saturation. 


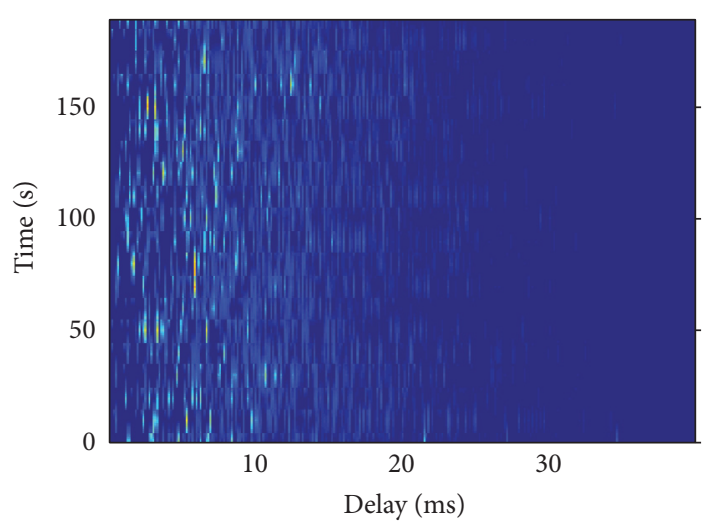

(a) $v_{11}$

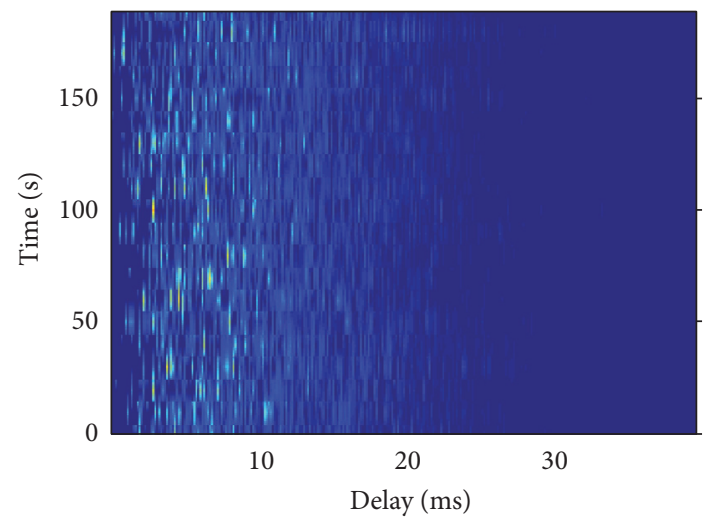

(c) $v_{21}$

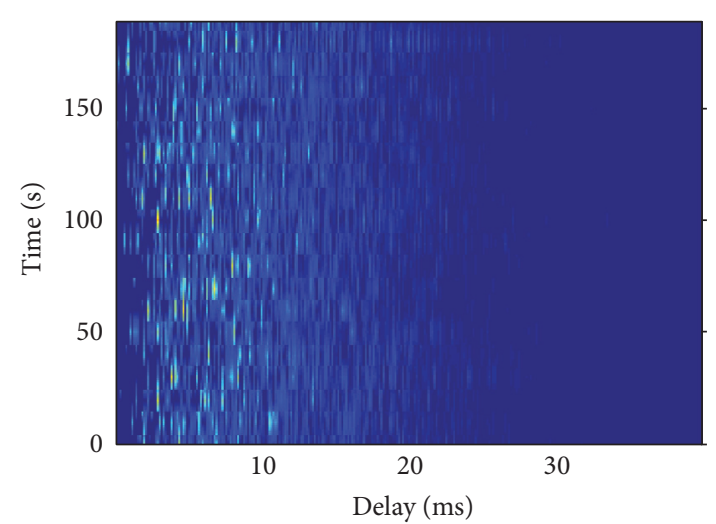

(b) $v_{12}$

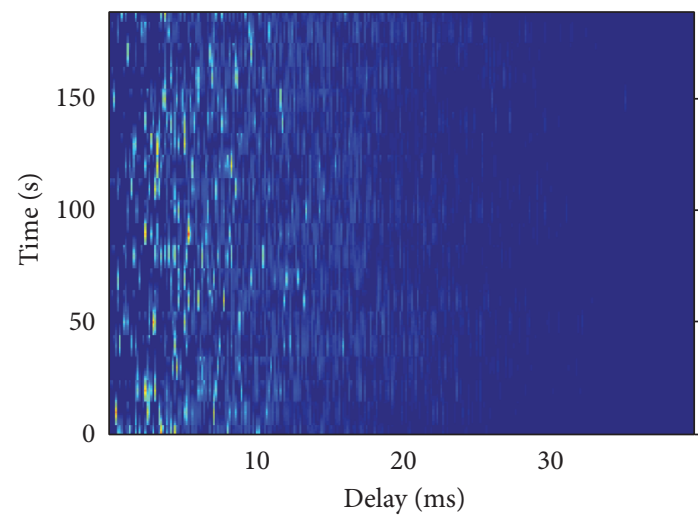

(d) $v_{22}$

FIGURE 4: The equivalent channel filter in amplify-and-forward underwater acoustic cooperative communication.

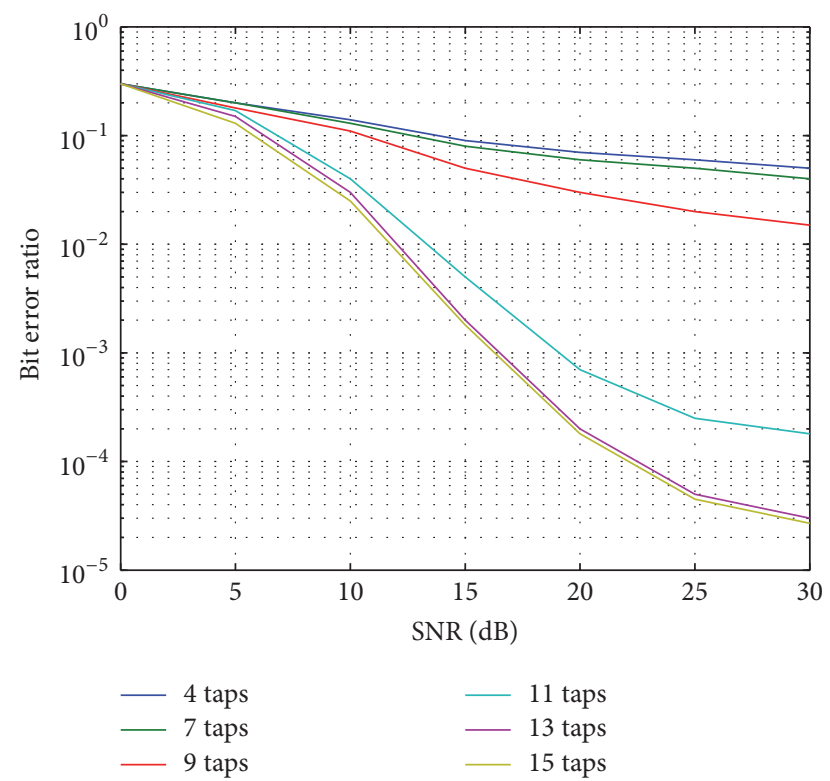

FIGURE 5: Bit error ratio with respect to different numbers of taps.

Comparison of the proposed time-reverse-and-forward underwater acoustic cooperative communication method to the existing underwater acoustic cooperative communication method is shown in Figure 6. From Figure 6, it can be seen that the same frequency bidirectional amplify-and-forward underwater cooperative method applied in [10] has poor performance. It requires $25 \mathrm{~dB}$ to keep the bit error ratio below $10^{-3}$. That is attribute to the cochannel interference and severe multipath as shown in Figure 4. The frequency division cooperative transmission method applied in [8] requires $18 \mathrm{~dB}$ to keep the bit error ratio below $10^{-3}$. Because of the complexity of underwater acoustic channel and the mutual interference to adjacent frequency bands, the performance is still unsatisfactory. Both the time division cooperative transmission method applied in [9] and MIMO signal processing applied in [6] show superiority in the bit error ratio performance that the bit error ratio reaches $10^{-3}$ at about $17 \mathrm{~dB}$. The performance of the proposed time-reverse-and-forward underwater acoustic cooperative communication method shows a little more progress, since multipath interference is compressed through time-reverse processing, while residue self-information interference is eliminated by additional cancelation. Moreover, the other virtue reflects in the simultaneous exchange of information between those two user nodes.

Sparsity of underwater acoustic channel is often large in deep sea and calm sea state, while it may be weaker in coastal waters or rough sea state. To this end, a more complex channel 


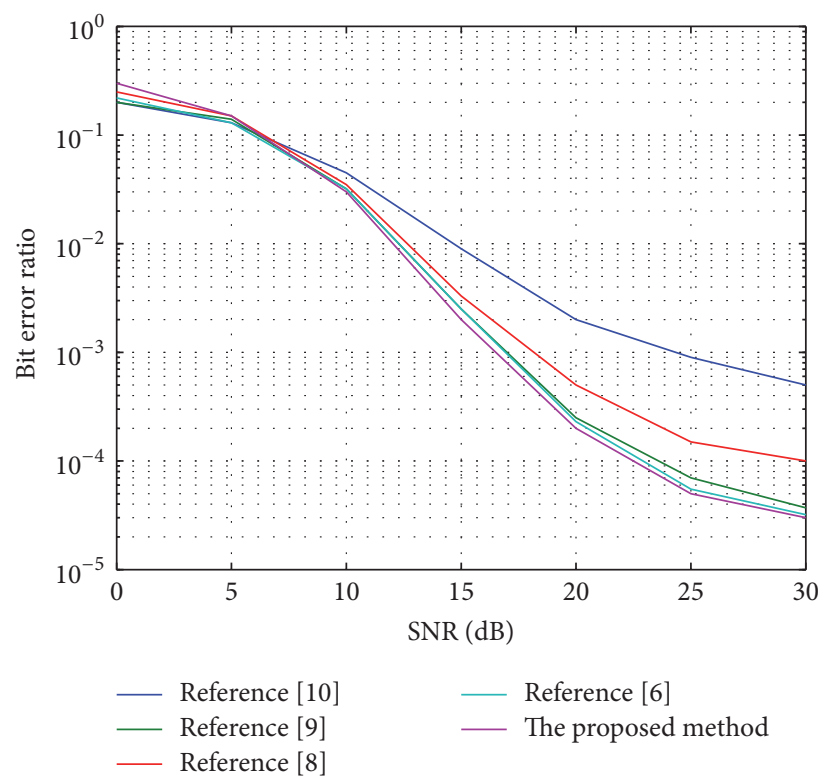

FIGURE 6: Bit error ratio over sparse channel with respect to different signal to noise ratio.

TABLE 1: Comparison of transmission rate.

\begin{tabular}{lcccc}
\hline SNR $(\mathrm{dB})$ & The proposed method & TDMA & FDMA & MIMO \\
\hline 5 & 1.7 & 0.85 & 0.85 & 0.87 \\
10 & 1.94 & 0.968 & 0.965 & 0.968 \\
15 & 1.992 & 0.997 & 0.996 & 0.997 \\
20 & 1.9996 & 0.9998 & 0.9995 & 0.9998 \\
\hline
\end{tabular}

is adopted to validate the performance of the proposed timereverse-and-forward underwater acoustic cooperative communication method. The maximum time delay is $60 \mathrm{~ms}$, with random numbers of paths within 30 to 60 , and the amplitude follows Rayleigh distribution. The resulting bit error ratio is shown in Figure 7. From Figure 7, it can be seen that all the existing cooperative communication methods show $2-3 \mathrm{~dB}$ degradation compared to that in Figure 6, since the multipath effect is more severe in complex channel. The proposed time-reverse-and-forward underwater acoustic cooperative communication method shows about $0.5 \mathrm{~dB}$ degradation compared to that in Figure 6, because of the adaption of timereverse processing to different channel condition.

5.3. Transmission Rate Analysis. Signal transmission over underwater acoustic channel suffers from the frequency dependant attenuation. Thus, the bandwidth of underwater acoustic channel is severely limited. Therefore, how to utilize the limited bandwidth of the underwater acoustic channel efficiently to provide high transmission rate plays a key role in design of the cooperative communication. Comparison of the transmission rate of the proposed time-reverse-and-forward underwater acoustic cooperative communication method to that of the existing cooperative method is shown in Table 1.

In Table 1, it can be seen that when the signal to noise ratio is over $15 \mathrm{~dB}$, the transmission rate of the proposed

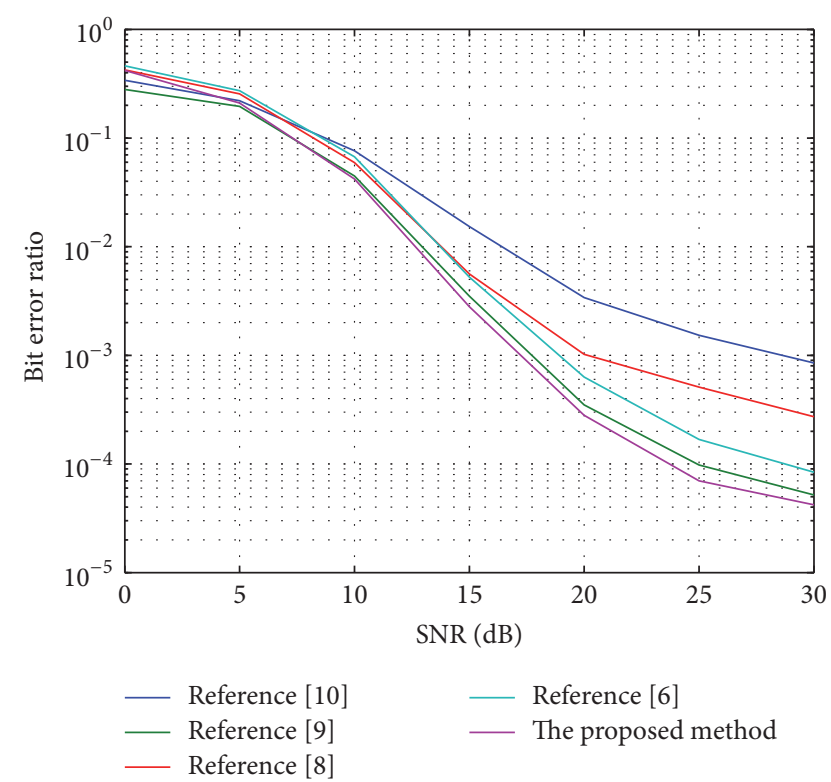

FIGURE 7: Bit error ratio over complex channel with respect to different signal to noise ratio.

time-reverse-and-forward bidirectional underwater acoustic cooperative communication method is about two times of that of the existing underwater acoustic cooperative communication methods. That is credited to the simultaneous bidirectional transmission and the efficient self-information interference cancelation.

\section{Conclusion}

Communications over underwater acoustic channel suffer from the strong multipath interference and great attenuation; thus it is hard to realize long range transmission. Underwater cooperative transmission has been regarded as the optimal solution. However, only single transmission is allowed in existing underwater acoustic cooperative transmission that limited the transmission rate. To solve this problem, a time-reverse-and-forward bidirectional underwater acoustic cooperative communication based on OFDM is investigated. Common uplink and downlink frequency bands are shared by all user nodes, and the received signal at the base station is time-reverse-and-forward to all user nodes without separation. Moreover, self-signal backpropagation is isolated at the received end to eliminate the self-information interference. Comparison from the respect of channel interference, bit error ratio, and transmission rate reveal that the proposed method is superior to the existing underwater cooperative transmission method. Specifically, the transmission rate of the proposed method is near double of the existing method when the required bit error ratio is no more than $10^{-3}$ and the signal to noise ratio is over $16.5 \mathrm{~dB}$.

\section{Competing Interests}

The authors declare that they have no competing interests. 


\section{Acknowledgments}

This work was supported by the National Natural Science Foundation of China (nos. 61531015 and 61471298).

\section{References}

[1] Y. Luo, L. Pu, H. Mo, Y. Zhu, Z. Peng, and J. Cui, "Receiver-initiated spectrum management for underwater cognitive acoustic network," IEEE Transactions on Mobile Computing, vol. 16, no. 1, pp. 198-212, 2017.

[2] F. Yuan, Q. Wei, and E. Cheng, "Joint virtual time reversal communications with an orthogonal chirp spread spectrum over underwater acoustic channel," Applied Acoustics, vol. 117, pp. 122-131, 2017.

[3] H. Kaushal and G. Kaddoum, "Underwater optical wireless communication," IEEE Access, vol. 4, no. 12, pp. 1518-1547, 2016.

[4] M. G. Tiquio, N. Marmier, and P. Francour, "Management frameworks for coastal and marine pollution in the European and South East Asian regions," Ocean \& Coastal Management, vol. 135, pp. 65-78, 2017.

[5] B. Peng and H. Dong, "DSP based real-time single carrier underwater acoustic communications using frequency domain turbo equalization," Physical Communication, vol. 18, pp. 40-48, 2016.

[6] L. Zhang, J. Huang, J. Han, and Q. Zhang, "SFICEE (spatialfrequency iterative channel estimation and equalization) in underwater acoustic MIMO-OFDM communication," Journal of Northwestern Polytechnical University, vol. 34, no. 2, pp. 208214, 2016.

[7] S. Liu and A. Song, "Optimization of LDPC codes over the underwater acoustic channel," International Journal of Distributed Sensor Networks, vol. 3, no. 6, pp. 1-6, 2016.

[8] J. Zhang, L.-L. Yang, L. Hanzo, and H. Gharavi, "Advances in cooperative single-carrier FDMA communications: beyond LTE-advancedS," IEEE Communications Surveys \& Tutorials, vol. 17, no. 2, pp. 730-756, 2015.

[9] F. Yang, Z. Lin, S. Zou, and Y. Tang, "A TDMA-based cooperative MAC protocol for vehicular ad hoc networks," Journal of Computational Information Systems, vol. 11, no. 10, pp. 35873594, 2015.

[10] R. Cao, F. Qu, and L. Yang, "Asynchronous amplify-and-forward relay communications for underwater acoustic networks," IET Communications, vol. 10, no. 6, pp. 677-684, 2016.

[11] M. V. Jamali, F. Akhoundi, and J. A. Salehi, "Performance characterization of relay-assisted wireless optical CDMA networks in turbulent underwater channel," IEEE Transactions on Wireless Communications, vol. 15, no. 6, pp. 4104-4116, 2016.

[12] Y. Li, Z. Jin, X. Wang, and Z. Liu, "Relay selection and optimization algorithm of power allocation based on channel delay for UWSN," International Journal of Future Generation Communication and Networking, vol. 9, no. 2, pp. 103-112, 2016.

[13] A. Salim and T. M. Duman, "A delay-tolerant asynchronous two-way-relay system over doubly-selective fading channels," IEEE Transactions on Wireless Communications, vol. 14, no. 7, pp. 3850-3865, 2015.

[14] Y. Zhang, Y. Chen, S. Zhou, X. Xu, X. Shen, and H. Wang, "Dynamic node cooperation in an underwater data collection network," IEEE Sensors Journal, vol. 16, no. 11, pp. 4127-4136, 2016.
[15] H. Nouri, M. Uysal, and E. Panayirci, "Information theoretical performance analysis and optimisation of cooperative underwater acoustic communication systems," IET Communications, vol. 10, no. 7, pp. 812-823, 2016.

[16] D. Zhao, J. Wen, and J. Si, "A UEP LT codes design with feedback for underwater communication," Journal of Sensors, vol. 2016, Article ID 2390734, 8 pages, 2016. 


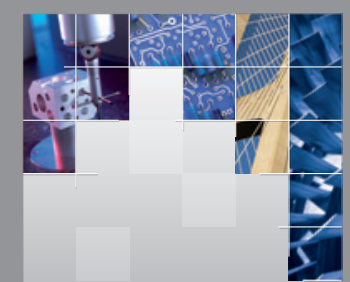

\section{Enfincering}
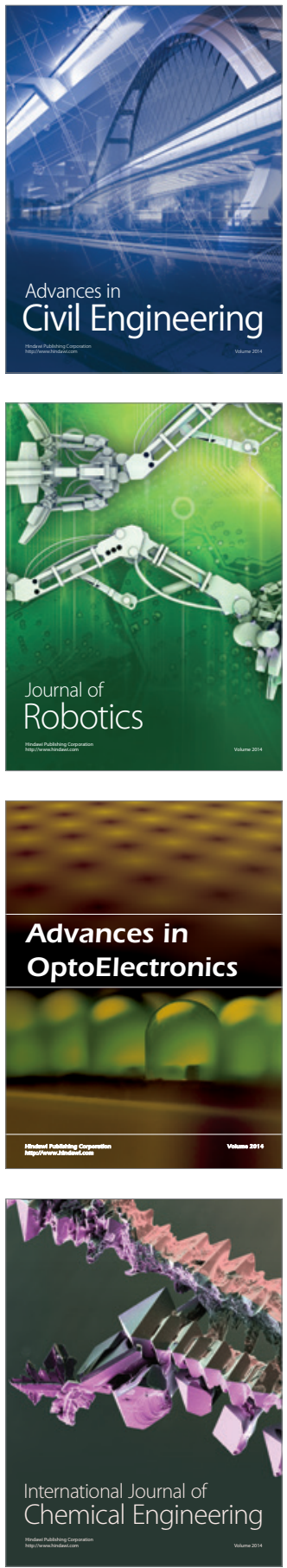

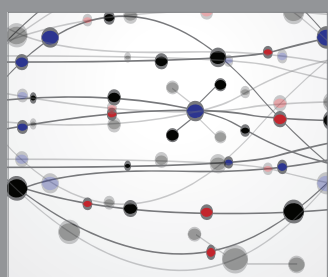

The Scientific World Journal

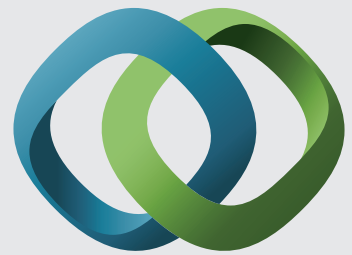

\section{Hindawi}

Submit your manuscripts at

https://www.hindawi.com
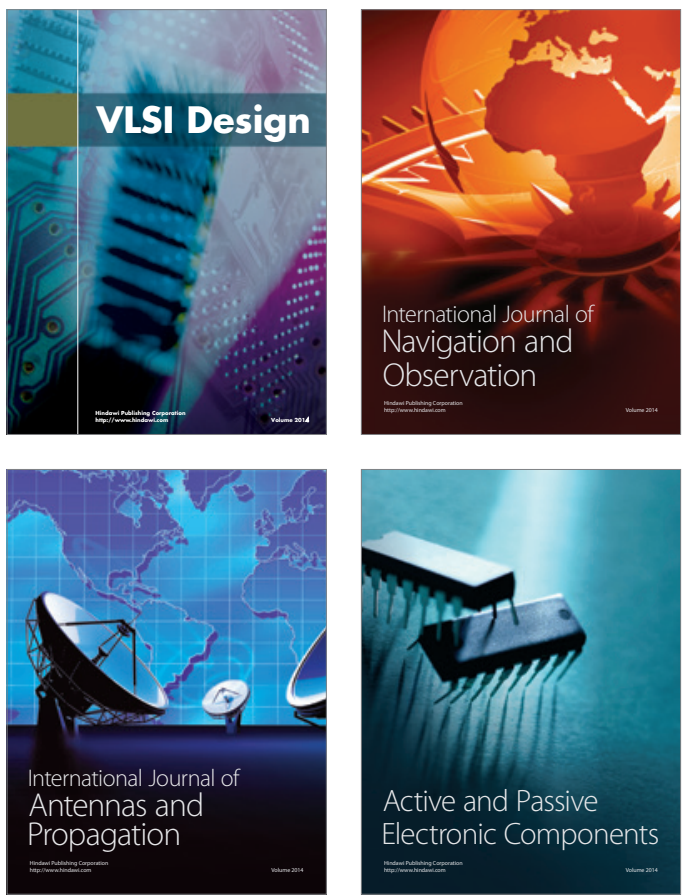
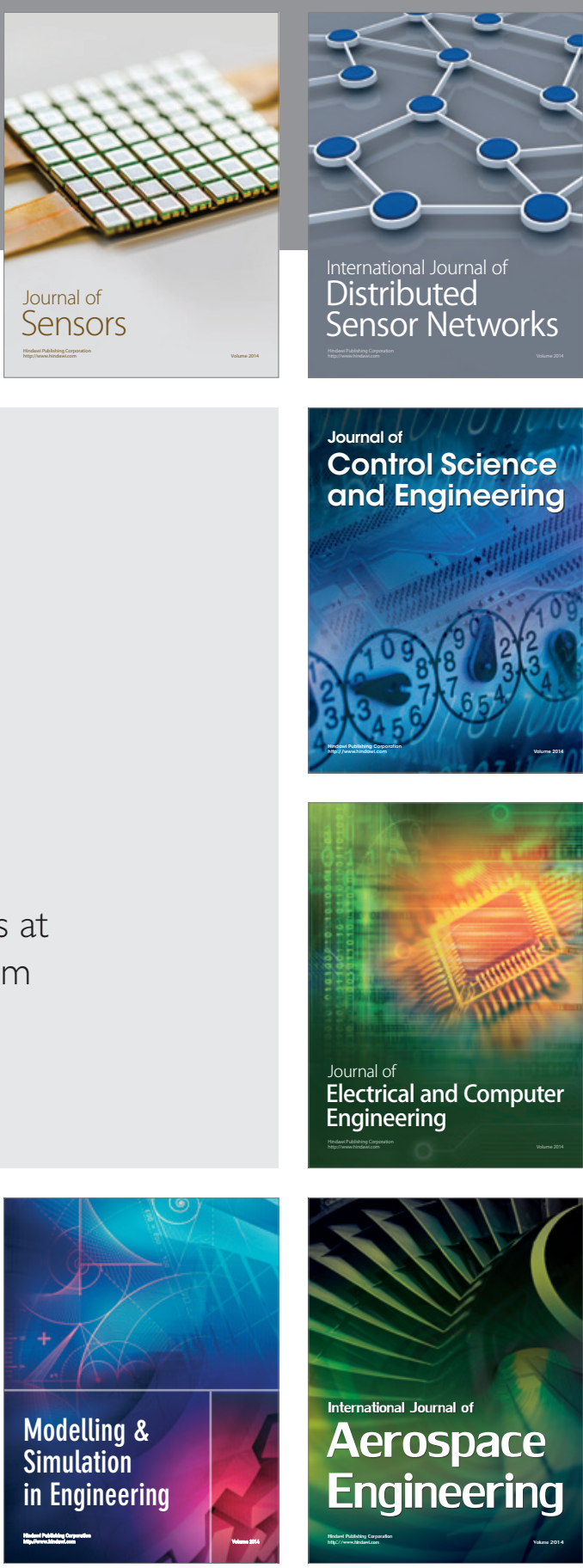

International Journal of

Distributed

Sensor Networks

$-$

Joumal of

Control Science

and Engineering
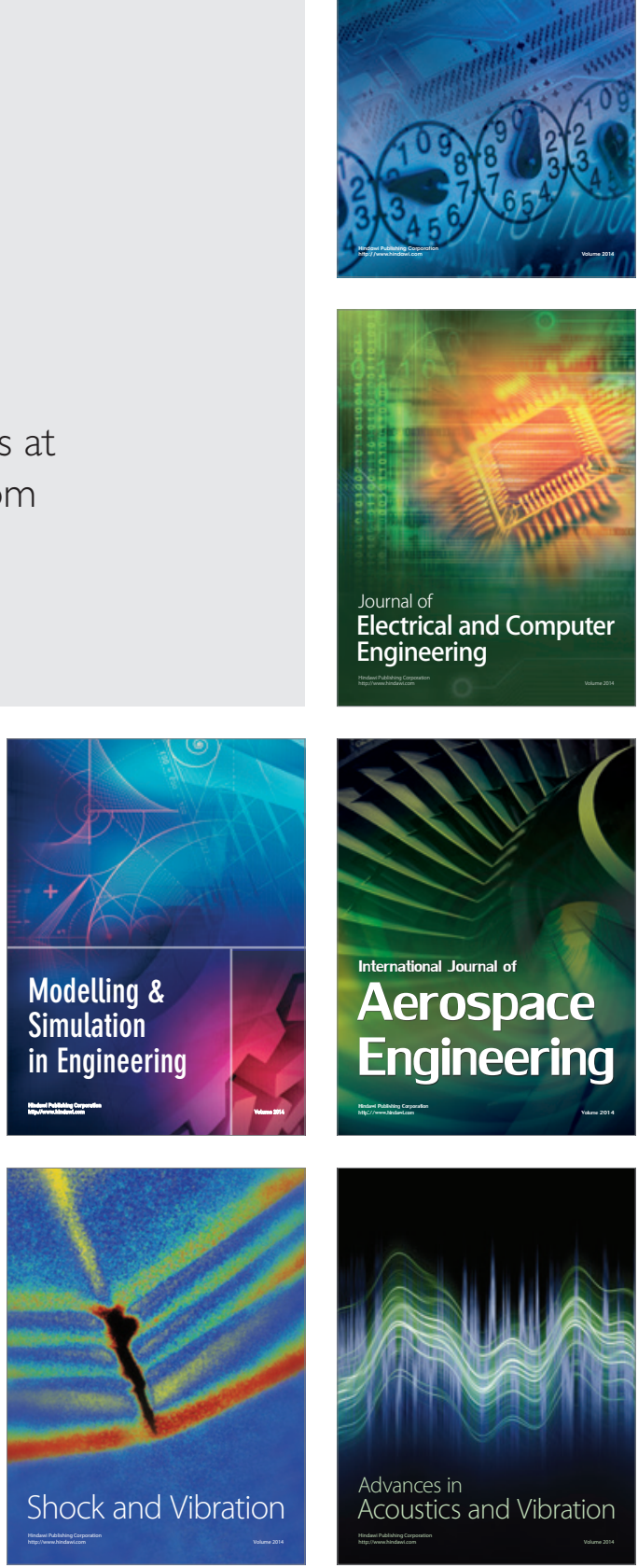